\title{
ARTICLE
}

Received 19 Jun 2012 | Accepted 5 Nov 2012 | Published 11 Dec 2012 DOl: 10.1038/ncomms2259

\section{Distinct modes of DNA accessibility in plant chromatin}

Huan Shu', Thomas Wildhaber ${ }^{1}$, Alexey Siretskiy², Wilhelm Gruissem¹ \& Lars Hennig1,2

The accessibility of DNA to regulatory proteins is a major property of the chromatin environment that favours or hinders transcription. Recent studies in flies reported that H3K9me2marked heterochromatin is accessible while H3K27me3-marked chromatin forms extensive domains of low accessibility. Here we show that plants regulate DNA accessibility differently. H3K9me2-marked heterochromatin is the least accessible in the Arabidopsis thaliana genome, and H3K27me3-marked chromatin also has low accessibility. We see that very long genes without $\mathrm{H} 3 \mathrm{~K} 9 \mathrm{me} 2$ or $\mathrm{H} 3 \mathrm{~K} 27 \mathrm{me} 3$ are often inaccessible and generated significantly lower amounts of antisense transcripts than other genes, suggesting that reduced accessibility is associated with reduced recognition of alternative promoters. Low accessibility of H3K9me2marked heterochromatin and long genes depend on cytosine methylation, explaining why chromatin accessibility differs between plants and flies. Together, we conclude that restriction of DNA accessibility is a local property of chromatin and not necessarily a consequence of microscopically visible compaction.

\footnotetext{
${ }^{1}$ Department of Biology, Plant Biotechnology, ETH Zurich, 8046 Zurich, Switzerland. ${ }^{2}$ Department of Plant Biology and Forest Genetics, Uppsala BioCenter Swedish University of Agricultural Sciences, Science for Life Laboratory and Linnean Center for Plant Biology, 75007 Uppsala, Sweden. Correspondence and requests for materials should be addressed to L.H. (email: lars.hennig@slu.se).
} 
$\mathrm{n}$ eukaryotes, DNA is wrapped around histone octamers forming the basic 'beads-on-a-string' structure of nucleosome arrays of chromatin. This $10 \mathrm{~nm}$ nucleosomal fiber can assemble into various higher-order structures. Chromatin organization differs greatly between genome regions, and mechanisms that cause local differences in chromatin include, among others, histone modifications, the insertion of histone variants, and nucleosome mobilization by chromatin-remodelling complexes ${ }^{1-3}$. A wellcharacterized type of chromatin is pericentromeric heterochromatin, which is typically enriched for repetitive sequences and transposable elements (TEs) and remains cytologically condensed even during interphase ${ }^{4}$. Histones in pericentromeric heterochromatin are hypoacetylated and enriched for methylation at lysine 9 of histone $\mathrm{H} 3$ (H3K9me2/3). Another well-characterized chromatin state is established by polycomb group ( $\mathrm{PcG}$ ) proteins, which are major regulators of developmentally controlled gene expression in animals and plants ${ }^{5,6}$. Polycomb repressive complex 2 (PRC2) tri-methylates $\mathrm{H} 3 \mathrm{~K} 27$, which in turn assists other PcG protein complexes such as PRC1 to introduce additional histone modifications. H3K27me3 often forms extensive domains in animals, but $\mathrm{H} 3 \mathrm{~K} 27 \mathrm{me} 3$ regions are largely restricted to singlegene bodies in Arabidopsis ${ }^{7-9}$.

Cytological identification of stably condensed chromatin has led to the assumption that condensed chromatin is static and prevents exchange of chromatin components and access of proteins to DNA. The view of higher-order structure as a regulator of accessibility, however, has been challenged by measurements of diffusional motilities of nuclear proteins. Proteins can readily diffuse even into highly condensed heterochromatin ${ }^{10-12}$, and inert diffusion probes of the size of large protein complexes readily entered condensed chromatin ${ }^{13,14}$. Similarly, a recent study of genome-wide DNA accessibility in Drosophila using M.SssI methylation footprinting questions the view of greatly reduced accessibility of heterochromatin: DNA in chromatin carrying $\mathrm{H} 3 \mathrm{~K} 9 \mathrm{me} 2$ seems to be as accessible as the rest of the genome ${ }^{15}$. Thus, higher-order folding and microscopically visible compaction of chromatin per se do not prevent access by nuclear proteins. It is possible that accessibility is mainly controlled locally by interactions of neighboring nucleosomes ${ }^{16}$. It is of great importance to discover determinants of DNA accessibility to nuclear proteins and the relation to cytological chromatin compaction.

In the model plant $A$. thaliana, cytologically condensed heterochromatin is considered to be of low complexity mostly comprising centromeric and pericentromeric sequences enriched in H3K9me2 and 5-cytosine DNA methylation ${ }^{17}$. We exploited this particular chromatin architecture to profile genome-wide DNA accessibility using DNase I sensitivity assays coupled to genome tiling microarrays. In contrast to the situation in Drosophila, the least accessible regions in the Arabidopsis genome were pericentromeric heterochromatin marked by $\mathrm{H} 3 \mathrm{~K} 9 \mathrm{me} 2$ and DNA methylation. Euchromatic and gene-dense chromosome arms were generally accessible but interrupted by isolated, inaccessible chromatin islands that lacked H3K9me2. Instead, these regions often comprised PcG protein targets or very long genes with high genic DNA methylation. Although DNA accessibility was generally negatively correlated with transcription, the group of very long and DNase I inaccessible genes were inaccessible despite being transcribed. As reduced accessibility of pericentromeric heterochromatin and long inaccessible genes required DNA methylation, it appears that PcG proteins and DNA methylation establish inaccessible chromatin in plants.

\section{Results}

Pericentromeric chromatin forms inaccessible regions. Differential accessibility of different chromosomal regions is not well understood. To better understand genomic DNA accessibility, we profiled global DNA inaccessibility in $A$. thaliana using chromatin DNase I hypo-sensitivity as a functional read-out. The rationale for our approach was that limited digestion with DNase I will generate small DNA fragments from accessible chromosome regions and larger fragments from less accessible regions, allowing separation by size-fractionation methods. Large DNA fragments were labelled and hybridized to genomic DNA tiling arrays (Fig. 1; see Methods section for details). To validate outcomes of this method, we compared microarray-derived inaccessibility scores to results of independent DNase I-PCR experiments without size fractionation for genomic fragments at two pericentromeric TE genes, two active genes and twelve fragments at genes of high inaccessibility (Supplementary Fig. S1). DNase I-PCR and DNase I-chip-derived DNA inaccessibility values agreed well, demonstrating the applicability of the arraybased profiling method.

DNA accessibility over the genome exhibited a wide dynamic range between the least and most accessible sites, and forms a continuous distribution with a pronounced tail toward high inaccessibility (Fig. 2a). We define the chromatin regions that are least sensitive to DNase I as 'inaccessible regions'. We identified 388 extended inaccessible regions of a total length of over $22 \mathrm{Mb}$. Conversely, we also identified 1957 hyper-accessible regions of a total length of $350 \mathrm{~kb}$ (see Methods). The hyperaccessible and inaccessible regions contained contrasting sets of genomic features (protein-coding genes, pseudogenes, TE genes, TEs; classification according to the Arabidopsis genome annotation) (Fig. 2b). The inaccessible regions comprised $\sim 17,600$ annotated genomic features, of which about $90 \%$ were TE genes, TEs and pseudogenes, and only a minor fraction $(<10 \%)$ were protein-coding genes. In contrast, the hyper-accessible regions comprised almost exclusively protein-coding genes (98\%) (Fig. 2b). Consistently, the density of inaccessible regions across the genome correlated positively with the TE density (Spearman's correlation per chromosome $=0.90 \pm 0.0, \quad P$-value $=1$ $\times 10^{-16}$ ) but negatively with protein-coding gene density (Spearman's correlation per chromosome $=-0.86 \pm 0.04$, $P$-value $\left.=1 \times 10^{-16}\right) \quad($ Fig. $2 c ; \quad$ Supplementary Fig. S2). Conversely, a majority $(\sim 93 \%)$ of permanently silenced TE genes reside in highly inaccessible chromatin. Notably, the $22 \mathrm{Mb}$ inaccessible regions comprised about $16 \%$ of the Arabidopsis genome $\left(\sim 135 \mathrm{Mb}^{18}\right)$, which closely matches results from previous cytological studies that estimated a chromocenter-localized heterochromatin fraction of $15-17 \%$ in Arabidopsis ${ }^{19,20}$. These results identify pericentromeric heterochromatin as a major component of inaccessible chromatin in Arabidopsis.

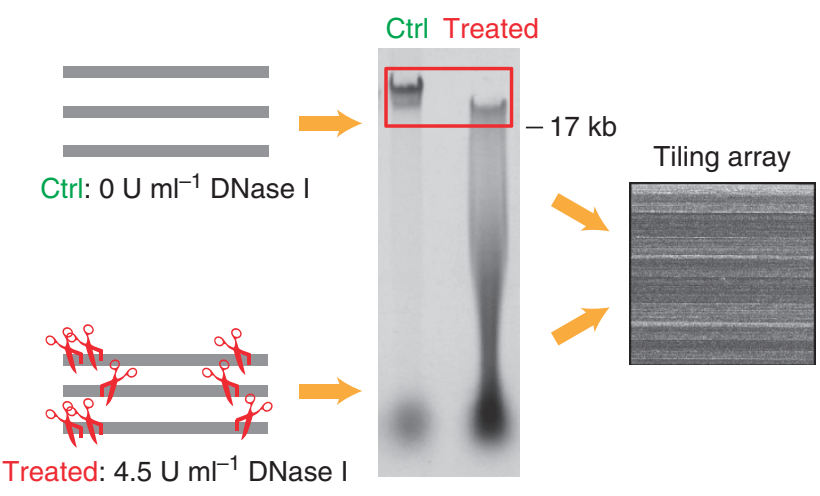

Figure 1 | Profiling DNA accessibility by DNase I-chip. Chromatin was partially digested with DNase I. Digested (red) and control (green) DNA fragments were hybridized onto microarrays. 


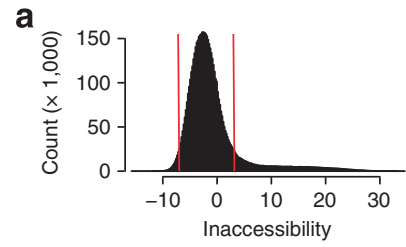

b

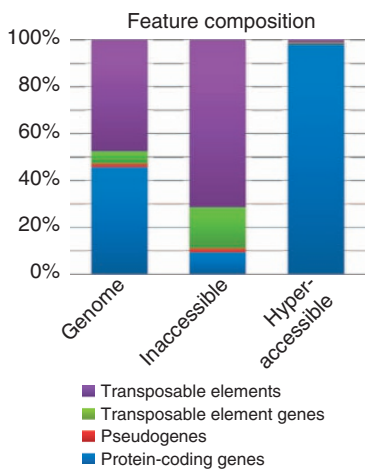

C
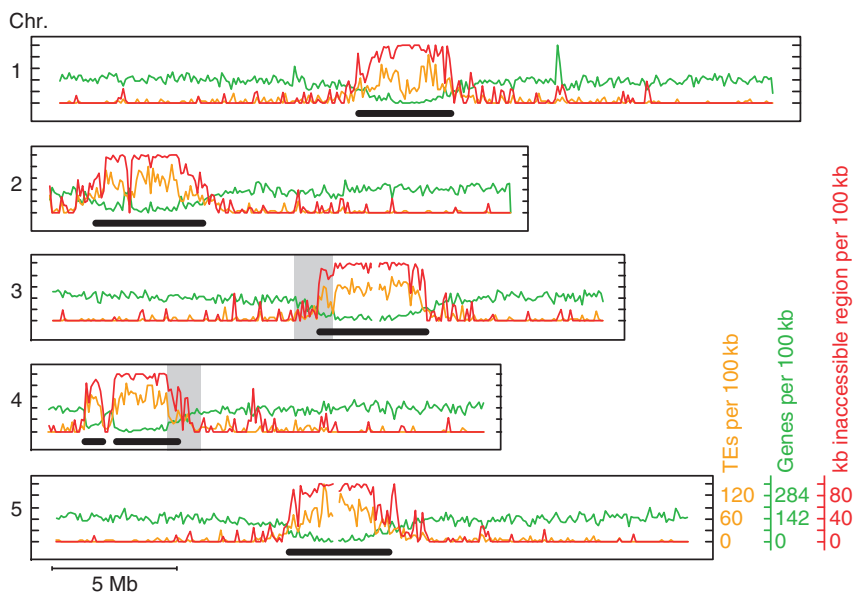

d
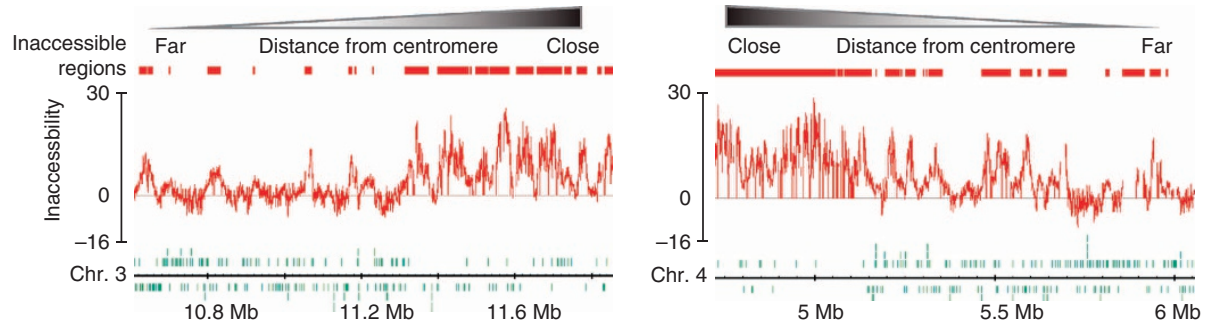

Figure 2 | Pericentromeric heterochromatin is least accessible. (a) Distribution of DNA inaccessibility scores. Vertical lines represent thresholds for the selection of hyper- and hypo-accessible regions. (b) Distribution of genomic features in inaccessible and hyper-accessible regions. (c) Density of inaccessible regions (red), protein-coding genes (green) and TEs (yellow) along chromosomes. Bars mark centromeric and pericentromeric heterochromatin. (d) Details of inaccessibility profiles at borders of pericentromeric heterochromatin (shaded blocks in c). Green bars represent annotated genes.

The hetero-to-euchromatin transition is gradual. Traditionally, Arabidopsis heterochromatin regions were probed by fluorescence in situ hybridization experiments ${ }^{21,22}$, which have limited resolution and throughput. Here, we attempted to define the borders of pericentromeric heterochromatin by an original definition-condensed chromatin regions with limited accessibility $^{23}$ - using the DNA inaccessibility measures as a functional proxy. Interestingly, however, pericentromeric sequences (Fig. 2c, black bars) were highly enriched for inaccessible chromatin regions but did not form uniform inaccessible blocks. Rather than forming sharp, step-like borders, the transition between inaccessible pericentromeric heterochromatin and accessible euchromatin arms was gradual. Inaccessible regions were more often interrupted by accessible regions with increasing distance from centromeres (Fig. 2d). These increasing interruptions mirrored the gradual increase in protein-coding gene density and the gradual decrease in TE density (Fig. 2c). In other words, inaccessible pericentromeric heterochromatin in Arabidopsis is not demarcated by sharp borders but invades into neighbouring accessible sequences.

$\mathrm{H} 3 \mathrm{~K} 9 \mathrm{me} 2$ is a major modification of inaccessible chromatin. Arabidopsis pericentromeric regions are enriched for $\mathrm{H} 3 \mathrm{~K} 9 \mathrm{me} 2$ and DNA-methylation ${ }^{24,25}$. Contrasting to the findings in flies, where H3K9me2-marked heterochromatin is as accessible as the rest of the genome, the pericentromeric enrichment of Arabidopsis inaccessible regions in our data suggests that $\mathrm{H} 3 \mathrm{~K} 9 \mathrm{me} 2$ is associated with inaccessible chromatin in Arabidopsis. We, therefore, explored the relationship between DNA accessibility and $\mathrm{H} 3 \mathrm{~K} 9 \mathrm{me}$. Globally, DNA inaccessibility correlated strongly and positively with H3K9me2 (Fig. 3a; Spearman's correlation per chromosome $=0.60 \pm 0.02, P$-value $\left.=2.2 \times 10^{-16}\right)$. Nevertheless, some sequences were highly accessible despite high H3K9me2 coverage (cloud below the diagonal in Fig. 3a). Manual inspection of several of these regions revealed that they usually contain small TE genes that are embedded in accessible chromatin environments of active genes. Next, we explored the $\mathrm{H} 3 \mathrm{~K} 9 \mathrm{me} 2$ distribution in proximities of all genes dependent on their different inaccessibility levels, quantified as a gene-wise inaccessibility score (see Methods). Of note, genes with high inaccessibility scores can reside outside of inaccessible regions, which were defined as inaccessible chromatin domains of extended lengths, not including short stretches of inaccessible chromatin. We grouped all genes by increasing inaccessibility, and plotted for each bin an averaged $\mathrm{H} 3 \mathrm{~K} 9 \mathrm{me} 2$ profile in the gene proximities (Fig. 3b). Clearly, the least accessible genes showed substantial H3K9me2 enrichment (Fig. 3b, bin 10, pink) both at gene bodies and at the proximal flanking sequences. In contrast, more accessible genes lacked $\mathrm{H} 3 \mathrm{~K} 9 \mathrm{me} 2$. This indicates that $\mathrm{H} 3 \mathrm{~K} 9 \mathrm{me} 2$ is an important component of the least accessible chromatin in Arabidopsis, much in contrast to the situation in flies.

Interestingly, genes in the second least accessible bin generally lacked H3K9me2 (Fig. 3b, bin 9, purple and Supplementary Fig. S3). This indicates that accessibility can be reduced without 

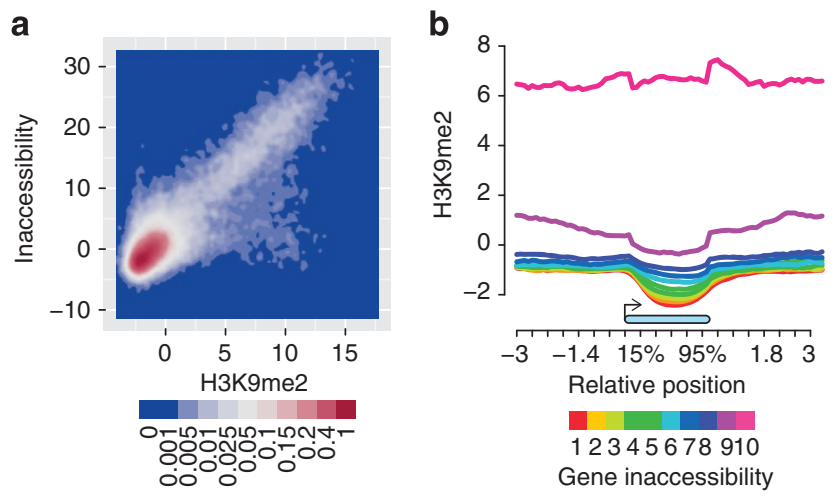

Figure 3 | Least accessible chromatin is rich in H3K9me2. (a) Distribution of DNA accessibility scores and H3K9me2 enrichments (red, high data point density; blue, low density). (b) H3K9me2 profiles across genes grouped by accessibility.

involving the canonical heterochromatic $\mathrm{H} 3 \mathrm{~K} 9 \mathrm{me} 2$. In contrast to the pericentromeric localization of most of the $\mathrm{H} 3 \mathrm{~K} 9 \mathrm{me} 2$-marked inaccessible genes, the inaccessible genes without $\mathrm{H} 3 \mathrm{~K} 9 \mathrm{me} 2$ were usually located in the inaccessible islands interspersed on the generich chromosome arms (Fig. 2c).

Together, we identified two major distinct types of inaccessible chromatin. The first type comprises pericentromeric heterochromatin and is associated with high levels of H3K9me2 and DNA methylation; the second type is scattered along the chromosome arms and does not carry H3K9me2.

Active genes are most accessible. DNA inaccessibility is thought to restrict recognition of DNA templates by regulatory proteins and thus to hinder transcription while accessible chromatin structures may facilitate transcription ${ }^{15,26}$. It is also possible that increased DNA accessibility is a consequence of transcriptional activity. In our data set, DNA accessibility and transcript levels had a positive correlation (Spearman's correlation per chromosome $=0.38 \pm 0.02, P$-value $\left.=2.2 \times 10^{-16}\right)$. The relation between transcript levels and DNA inaccessibility was gradual rather than step-like (Fig. 4a), suggesting that increased transcription correlates with a gradual increase in DNA accessibility and not with a switch-like, sharp increase. Next, we tested how far effects of transcription on DNA accessibility extend from the transcription unit. Averaged DNA inaccessibility profiles across genes were plotted after grouping genes into four bins of increasing transcript abundance (Fig. 4b). Bodies and flanking sequences of inactive genes showed high inaccessibility signals, suggesting that such genes often reside in neighbourhoods of low DNA accessibility (Fig. 4b, red, green, bins 1-2). In contrast, active genes had low inaccessibility signals (Fig. 4b, cyan, purple, bins 3-4). Notably, accessibility was considerably higher at gene bodies than at flanking sequences. This indicates that the increase of DNA accessibility associated with transcription acts locally and does not extend far from the transcription unit.

PcG targets have reduced accessibility. To identify mechanisms that further reduce accessibility of inactive genes, we next focused on PcG proteins, which form a major class of transcriptional repressors. PcG proteins are essential for normal development but the mechanisms by which they repress target gene transcription are not well understood. About two decades ago, it was proposed that animal PcG proteins repress transcription by chromatin compaction and reducing DNA accessibility, but only recently evidence supporting this view has started to accumulate for
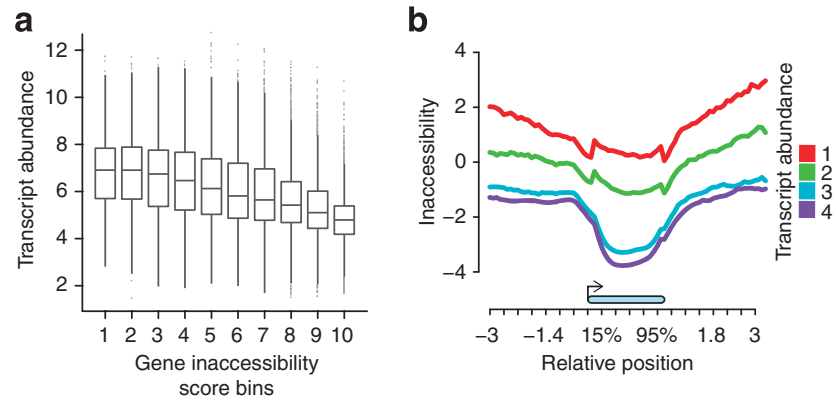

Figure 4 | Inactive genes are less accessible than active genes. (a) Relation between transcript abundance and DNA accessibility. (b) DNA accessibility profiles across genes grouped by expression.
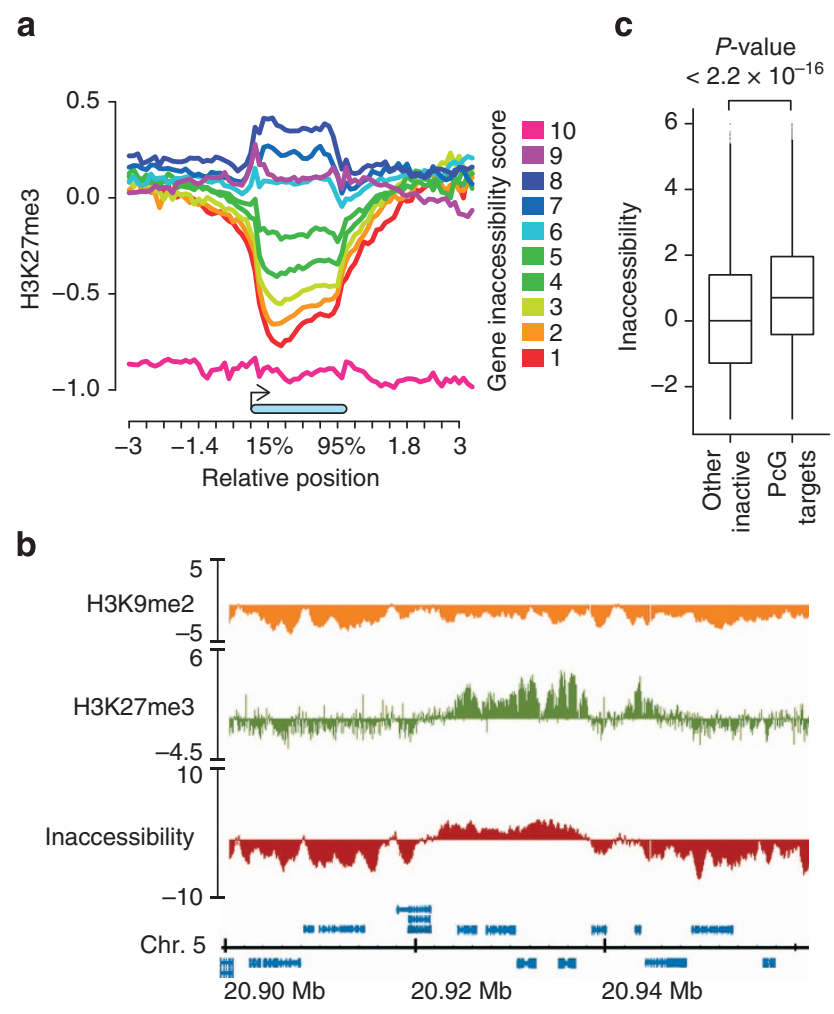

Figure 5 | Targets of Polycomb group proteins have low accessibility.

(a) H3K27me3 profiles across genes grouped by accessibility.

(b) Epigenome profiles along a region on chromosome 5 including several PcG target genes. (c) Inaccessibility of other inactive genes and PcG targets ( $P$-value was from Wilcoxon's-signed rank test, one-tail).

animal systems ${ }^{15,27-29}$. As patterns of H3K27me3 enrichment and PcG protein complex composition are only partially conserved between plants and animals ${ }^{5}$, it is not clear whether in plants repression by $\mathrm{PcG}$ proteins involves chromatin compaction. Here, we asked whether plant PcG target genes contributed to the set of inaccessible genes. Indeed, nearly half (46.2\%) of the genes in inaccessible regions without H3K9me2 were PcG targets. Levels of H3K27me3, the hallmark of PcG repression, showed positive correlation at gene level with DNA inaccessibility (Fig. 5a; see Fig. 5b for an example). Specifically, H3K27me3 was clearly enriched in bodies of genes with low accessibility. H3K27me3 was only partially enriched in the least accessible genes as this group is dominated by genes marked by 


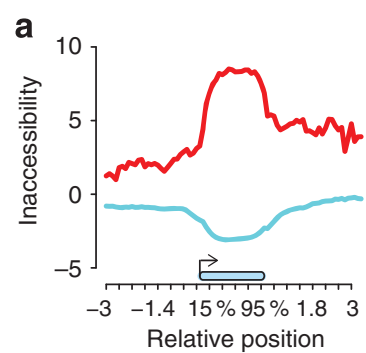

d
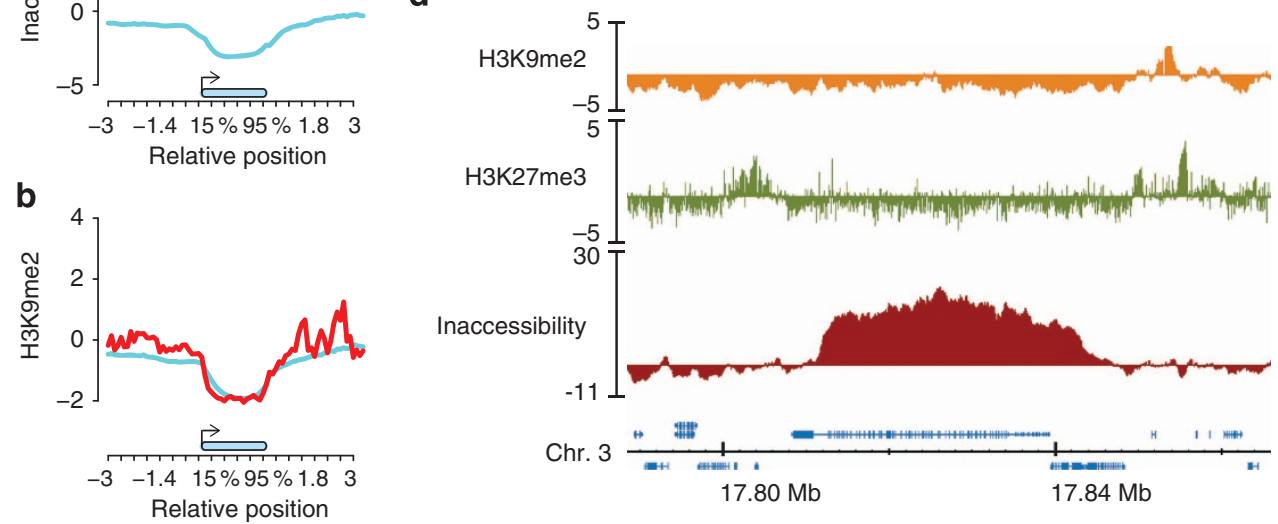

C

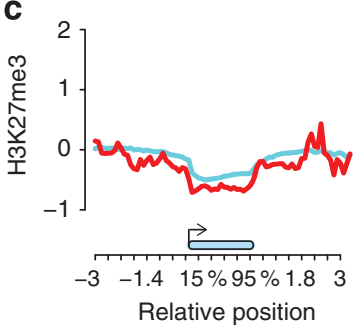

e

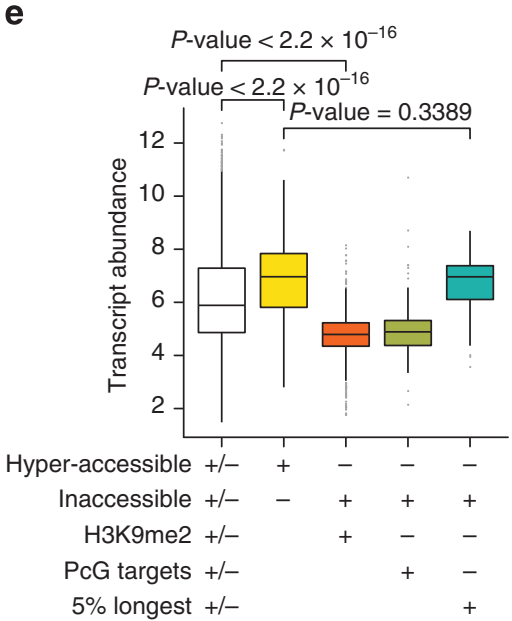

f
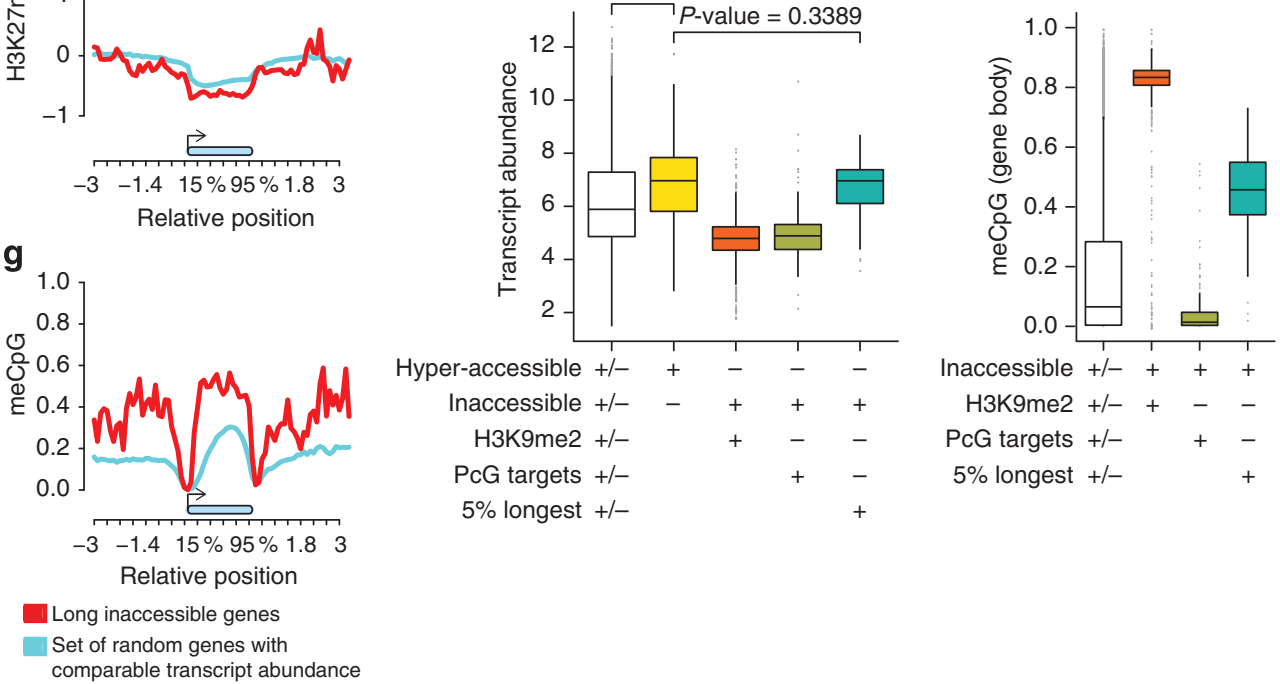

Figure 6 | The longest genes have low accessibility even when transcribed. (a-c, g) Inaccessibility, H3K9me2, H3K27me3 and methylated CpG (meCpG) profiles across long inaccessible genes (red) and accessible genes with similar sense transcript levels (azure). (d) Epigenome profiles along a region on chromosome 3 including a very-long gene. (e) Transcript abundance of genes grouped by accessibility ( $P$-values from Wilcoxon's-signed rank test, twotail). (f) Cytosine methylation in -Cytosine-phosphate-Guanine- $(\mathrm{CpG})$ contexts in gene bodies.

H3K9me2, which together with DNA methylation excludes H3K27me ${ }^{7,9,30}$. Notably, PcG targets were significantly less accessible than other inactive genes lacking H3K9me2 $(P$ value $\left.=2.2 \times 10^{-16}\right) \quad$ (Fig. $5 \mathrm{c}$ ), indicating that the reduced accessibility of the PcG targets is not a mere consequence of repressed transcription. These results reveal that gene repression by plant PcG proteins is associated with locally reduced DNA accessibility.

Long genes methylated in the gene body are inaccessible. In addition to the two classes of inaccessible genes defined by H3K9me2 or H3K27me3, we identified a third class, which carries neither H3K9me2 nor H3K27me3 (Fig. 6a-c; see Fig. 6d for an example). Genes in this class were often very long (Fig. 6d; Supplementary Fig. S4); nearly half of the inaccessible genes lacking both H3K9me2 and H3K27me3 belonged to the 5\% longest of all genes. We next investigated whether these long genes had low accessibility because of repressed transcription by comparing transcript levels of the different classes of inaccessible genes. Consistent with the global correlation between transcription and accessibility, inaccessible genes with $\mathrm{H} 3 \mathrm{~K} 9 \mathrm{me} 2$ or H3K27me3, and genes from hyper-accessible regions (hyperaccessible genes) had low and high transcript levels, respectively (Fig. 6e). Surprisingly, the long inaccessible genes were not silenced but expressed at levels comparable to the hyper-accessible genes (Fig. 6e), suggesting that this type of chromatin compaction is not associated with canonical repression of transcription. In addition, this observation demonstrates that transcription does not inevitably cause increased DNA accessibility.

It has been reported that long Arabidopsis genes are enriched for genic DNA methylation especially toward the $3^{\prime}$-end ${ }^{31-33}$. Confirming previous observations, cytosine methylation in CpG contexts in gene bodies of long inaccessible genes was significantly above the genome median $\left(P\right.$-value $\left.=2.2 \times 10^{-16}\right)$ but lower than methylation in inaccessible genes with $\mathrm{H} 3 \mathrm{~K} 9 \mathrm{me} 2$ 
$\left(P\right.$-value $=2.2 \times 10^{-16}$; Fig. $\left.6 \mathrm{f}-\mathrm{g}\right)$. Cytosine methylation was not increased in other sequence contexts or in promoters of these long inaccessible genes (Supplementary Fig. S5). DNA methylation in gene bodies but not promoters also coincided with higher DNA inaccessibility in gene bodies than in promoters (compare Fig. 6a,g). Thus, it appeared possible that DNA methylation on $\mathrm{CpG}$ nucleotides in gene bodies contributes to reduced DNA accessibility.

Inaccessibility of TE and long genes requires DNA methylation. We have found that $\mathrm{H} 3 \mathrm{~K} 9 \mathrm{me} 2$-marked pericentromeric heterochromatin is inaccessible in the Arabidopsis genome. This was in contrast to flies, where $\mathrm{H} 3 \mathrm{~K} 9 \mathrm{me} 2$-marked heterochromatin is not particular inaccessible. We, therefore, considered molecular mechanisms that could contribute to the low accessibility of H3K9me2-marked chromatin in Arabidopsis. As both $\mathrm{H} 3 \mathrm{~K} 9 \mathrm{me} 2$-marked genes and long inaccessible genes were highly methylated at cytosines ${ }^{25,32}$ (Fig. 6g,f; Supplementary Fig. S5) but DNA methylation is very low in flies ${ }^{34}$, we hypothesized that cytosine methylation contributes to low DNA accessibility in Arabidopsis. This hypothesis was tested by treating seedlings with 5-azacytidine, which is an established method to deplete the Arabidopsis genome of cytosine methylation ${ }^{35-37}$. In treated seedlings, accessibility of tested long genes and TE genes increased strongly and became similar to that of accessible active genes (Fig. 7a). Similarly, genetic reduction of cytosine methylation in a $d d m 1$ mutant $^{38-40}$ also caused increased accessibility of the tested fragments (Supplementary Fig. S6). Importantly, only one out of the six inaccessible genes tested showed increased transcript abundance upon 5-azacytidine treatment (Fig. 7b). Thus, the general increase in accessibility was not a mere consequence of transcription activation. These results indicate that cytosine methylation is required to reduce the accessibility of long genes and TE genes.

Inaccessible long genes have reduced antisense transcription. It has been proposed that genic DNA methylation could function to repress intragenic cryptic promoters ${ }^{31}$. We, therefore, considered that the reduced DNA accessibility mediated by $\mathrm{CpG}$ methylation at the long inaccessible genes is associated with repression of cryptic promoters in the gene body. We reasoned that antisense transcript levels could be used as proxy for the activity of alternative promoters and tested whether the low accessibility of the long genes was associated with low antisense transcript levels. Indeed, antisense transcript levels of long inaccessible genes were significantly below the genome average (Fig. 8a; $P$-value $\left.=5.5 \times 10^{-8}\right)$. This is in stark contrast to the abundance of sense transcripts, which were considerably above genome average (Fig. 6b). Inaccessible long genes had significantly lower antisense transcript levels than other long genes $\left(P\right.$-value $\left.=3.3 \times 10^{-6}\right) \quad$ (Fig. $8 \mathrm{~b}$, left). In contrast, inaccessible and accessible long genes had sense transcript levels not significantly different from each other ( $P$-value $=0.053$ ) (Fig. 8b, right). Therefore, the reduced accessibility seems to be preferentially associated with repression of transcription from alternative promoters. Identical results were also obtained using publically available strand-specific RNA sequencing data ${ }^{41}$ (Supplementary Fig. S7). Genome-wide, higher accessibility of genes also seems to allow more antisense transcription. For the hyper-accessible genes, not only levels of sense (Fig. 6b) but also antisense transcripts were significantly higher than the genome median $\left(P\right.$-value $=5.9 \times 10^{-5}$; Fig. $\left.8 \mathrm{a}\right)$. Together, these results suggest that transcription from intragenic promoters is not strongly controlled for most active genes but is reduced at long inaccessible genes.
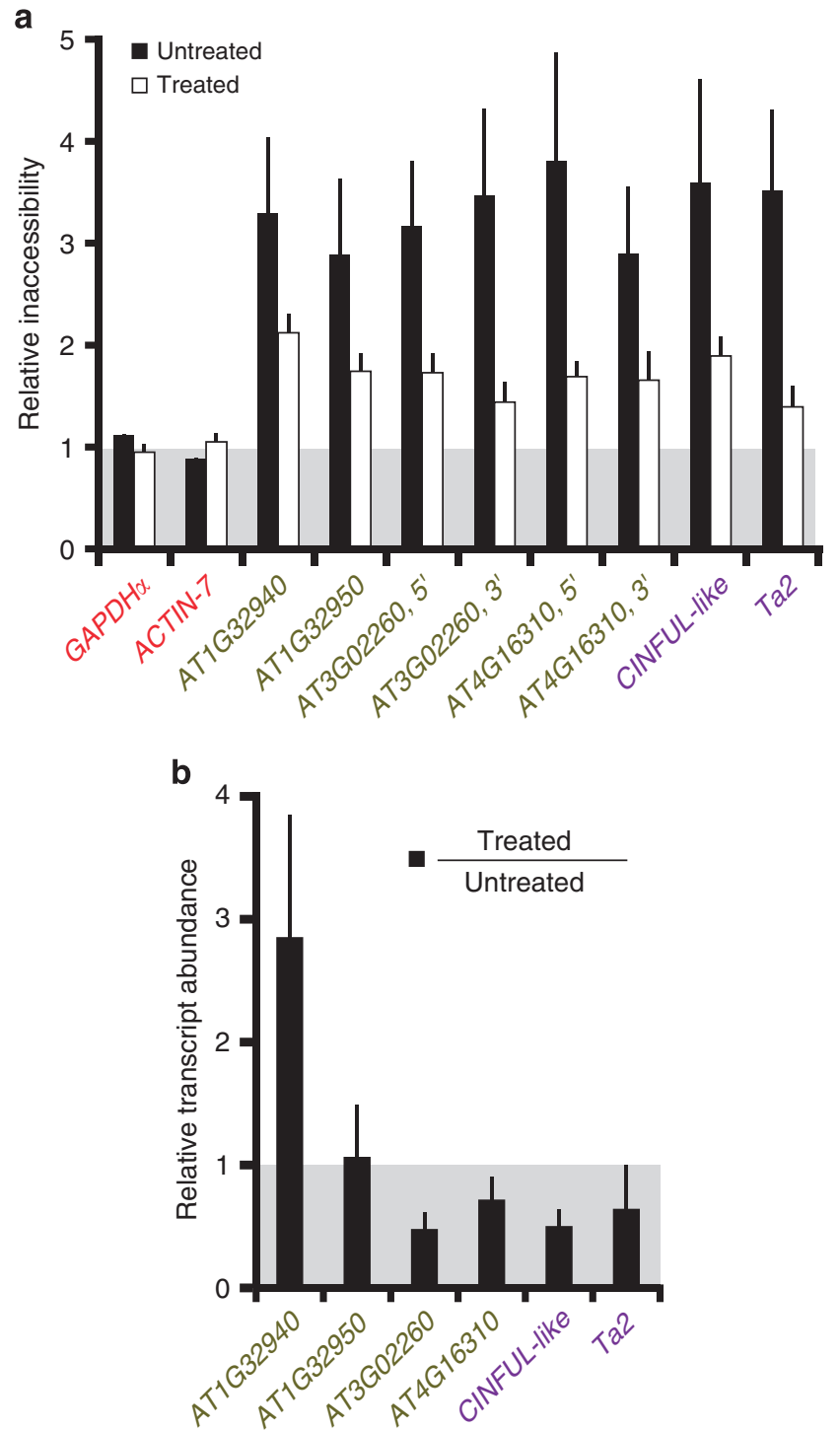

Figure 7 | Low accessibility of long genes requires DNA methylation. (a) DNA accessibility at accessible active (red), inaccessible long active (green) and inaccessible silent genes (purple) after treatment with 5-azacytidine to reduce DNA methylation. (b) Effect of 5-azacytidine treatment on transcript levels. (a) and (b) show means of three biological replicates. Error bars show s.e.m. a

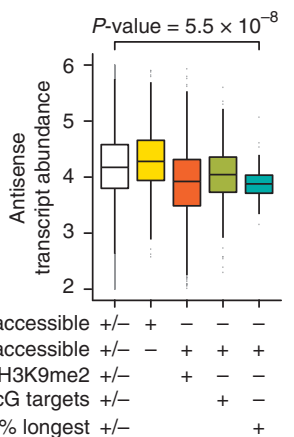

b

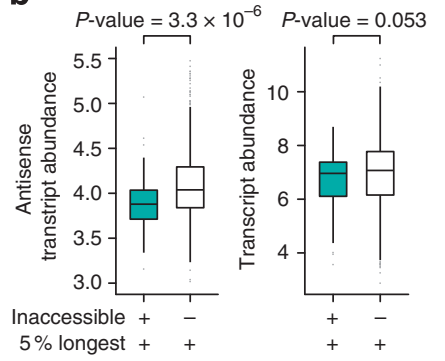

Figure 8 | Inaccessible long genes generate low levels of antisense transcripts. (a) Levels of antisense transcripts in genes grouped by accessibility. (b) Antisense and sense transcript levels in inaccessible and accessible long genes. P-values were from Wilcoxon's-signed rank test, one-tail. 


\section{Discussion}

DNA accessibility is thought to be important for the regulation of transcriptional control, and it has been proposed that a major function of the multiple combinations of histone modifications is to fine-tune accessibility of the $\mathrm{DNA}^{42}$. As constitutive heterochromatin contains mostly silent sequences that remain densely compacted even during interphase, ${ }^{4}$, it was generally thought to strongly restrict the access of proteins to DNA. However, several studies have now suggested that this view might be an oversimplification. Not only do various high-molecular weight probes and proteins readily enter heterochromatin ${ }^{10-14}$, but also transcription of repetitive sequences by specific RNA polymerases is required for methylation of $\mathrm{H} 3 \mathrm{~K} 9$ and heterochromatin assembly ${ }^{43}$. It is possible that bursts of transcription occur particularly during S-phase offering an explanation to reconcile the conflicting concepts of transcriptional repression and active transcription in heterochromatin ${ }^{44-46}$. More recently, the prevailing concept of constitutive heterochromatin being inaccessible was again challenged by the finding that heterochromatin in Drosophila was not less accessible than the genome average ${ }^{15}$. In stark contrast, our data revealed that H3K9me2-marked heterochromatin regions in Arabidopsis have by far the lowest DNase I sensitivity in the genome. In contrast to flies, where $\mathrm{H} 3 \mathrm{~K} 9 \mathrm{me} 2$ and $\mathrm{H} 3 \mathrm{~K} 9 \mathrm{me} 3$ are both enriched in constitutive heterochromatin; in Arabidopsis only $\mathrm{H} 3 \mathrm{~K} 9 \mathrm{me} 2$ is heterochromatic while $\mathrm{H} 3 \mathrm{~K} 9 \mathrm{me} 3$ is present in the euchromatin. Future work must address how H3K9me3 differentially affects fly heterochromatin and Arabidopsis euchromatin. In Arabidopsis but not in flies, $\mathrm{H} 3 \mathrm{~K} 9 \mathrm{me} 2$-marked heterochromatin is also rich in 5 -cytosine DNA methylation ${ }^{25}$. While we found that DNA methylation is required for low accessibility of several tested loci, DNA methylation was reported to be only partially required for formation of heterochromatic chromocenters ${ }^{19}$. These findings support the notion that microscopically visible compaction such as in heterochromatic chromocenters per se does not greatly restrict accessibility but that DNA accessibility is instead mostly defined at a lower organisational level of chromatin ${ }^{16}$. Therefore, compaction at a cytological scale as visible by microscopy should be differentiated from reduced accessibility, which is established locally and might act mostly at the scale of neighbouring nucleosomes.

Similar to repression of sequences in constitutive heterochromatin, silencing of genes targeted by $\mathrm{PcG}$ proteins was proposed to result in reduced accessibility. Indeed, long-distance chromatin contacts establish microscopically visible compaction of PcG target genes in Drosophila ${ }^{47}$, and in vitro experiments had suggested that fly PRC1 can compact nucleosome arrays ${ }^{48}$. More recently, it was reported that PcG protein targeting reduces DNA accessibility in Drosophila $^{15}$. In plants, the PcG protein system is partially conserved, but important differences to animals exist ${ }^{5}$. In particular, a strict homolog of PRC1 seems absent and PcG targeting does not usually manifest itself in patterns similar to the whole chromosome repression of Xi or long-range repression of coregulated gene clusters like Hox genes in animals. Instead, the H3K27me3 mark in Arabidopsis is typically restricted to singlegene bodies ${ }^{7-9}$. Thus, it was well possible that PcG proteinmediated silencing in plants involves different molecular mechanisms and did not involve chromatin compaction. Our DNA inaccessibility data showed global positive correlation between $\mathrm{H} 3 \mathrm{~K} 27 \mathrm{me} 3$ levels and gene inaccessibility; however, thus, supporting a conserved mechanism of chromatin packaging by PcG proteins in plants and animals. An important question for future studies is whether and how the compact chromatin of the PcG targeted genes is controlled by the plant PcG protein complexes.

In addition to genes in constitutive heterochromatin and to PcG protein target genes, we identified a class of inaccessible genes that were very long and had high intragenic $\mathrm{CpG}$ methylation. Intragenic $\mathrm{CpG}$ methylation was previously found in both mammals ${ }^{49}$ and plants ${ }^{31-33}$. It has been proposed that genic DNA methylation results from occasional divergent transcription from cryptic promoters within the gene body that would recruit small interfering RNA-directed DNA methylation activity $^{31,33}$. This pathway is possibly distinct from formation of constitutive heterochromatin because CpG methylation in gene bodies is independent of $\mathrm{H} 3 \mathrm{~K} 9 \mathrm{me} 2$ (refs 31-33). Gene body $\mathrm{CpG}$ methylation could interfere with aberrant transcription by restricting access of RNA Polymerase II to intragenic cryptic promoters $^{31}$ or by interfering with elongation of RNAP II (ref. 33). It is possible that gene body $\mathrm{CpG}$ methylation interferes with transcription via chromatin compaction, possibly by recruiting histone deacetylases ${ }^{4}$ or by excluding nucleosomedestabilising histone variants ${ }^{50}$. We found in Arabidopsis that long inaccessible genes had lower antisense transcript levels and that DNA methylation was required for low accessibility. These results support a model in which intragenic CpG methylation leads to reduced DNA accessibility, which in turn represses alternative (or cryptic) promoters. Consistent with this notion, sequences at proximal promoters and in the most $5^{\prime}$-region of gene bodies were often excluded from the domain of low accessibility (Fig. 6a), suggesting that accessible promoters are needed to sustain transcription of regular transcripts.

Facilitating or restricting access to DNA is an ultimate functional output of chromatin, and genome-scale information on DNA inaccessibility is needed for the interpretation of epigenome profiling studies. Our data suggest a complex model of regulation of DNA accessibility. Inactive genes have an intermediate accessibility, which can be greatly reduced by repressive PcG proteins or heterochromatin components. Conversely, transcription is often associated with a local increase in accessibility. For some active genes, increased accessibility is restricted to promoters and $5^{\prime}$-sequences while the accessibility of the largest part of the gene body is strongly reduced by DNA methylation thus preventing transcription from alternative promoters. As DNA methylation was required for reduced accessibility of $\mathrm{H} 3 \mathrm{~K} 9$ me2-marked heterochromatic sequences, we propose that differences in genomic cytosine methylation cause different heterochromatin accessibility between Arabidopsis and Drosophila.

\section{Methods}

Plant material. All experiments used Arabidopsis thaliana accession Columbia-0 plants. Plants were grown on soil at $21^{\circ} \mathrm{C}$ in darkness $(16 \mathrm{~h})$ and at $20^{\circ} \mathrm{C}$ in light ( 8 h). Leaves (leaf no. 6 from $\sim 5$ plants per sample) were harvested after 35 days at zeitgeber time 7 (that is, $7 \mathrm{~h}$ after start of the photoperiod) and frozen in liquid nitrogen. Note that cell division and expansion had ceased in the harvested leaves. For experiments with reduced DNA methylation, seeds were sown on Murashige and Skoog (MS) plates containing $7 \mu \mathrm{g} \mathrm{ml}^{-1}$ of 5-azacytidine and grown under long day conditions for 14 days ${ }^{51}$.

DNase I-PCR. Nuclei from $200 \mathrm{mg}$ of plant material were prepared and treated as described in the Methods section. Five aliquots of $\frac{1}{8}$ of the extracted nuclei were subjected to DNase I digestion at final concentrations of $0,1,2,4$ and $8 \mathrm{Uml}^{-1}$ DNA was extracted from three aliquots of the nuclei using careful phenolchloroform extraction and ethanol/salt precipitation. Extracted DNA was redissolved in digestion buffer and digested at final DNase I concentrations of $0,0.25$ and $0.75 \mathrm{U} \mathrm{ml}^{-1}$. DNA recovered from either the digested nuclei or digested DNA was used as template for PCR using specific primers amplifying fragments of about $1.5 \mathrm{~kb}$ (Supplementary Table S1). PCR products were quantified using a DNA 7500 kit on the Lab-on-chip platform (Agilent, California), and relative amounts of each fragment were calculated by normalizing to the amount of PCR product from the $0 \mathrm{U} \mathrm{ml}^{-1}$ DNase I-treated samples. To compare the DNA accessibility in plant treated or not treated with 5-azacytidine, nuclei from $25 \mathrm{mg}$ of 14-day old seedlings were subjected to DNase I digestion at final concentrations of 0.05 or $2 \mathrm{U} \mathrm{ml}^{-1}$. PCR was performed as above. To calculate DNA inaccessibility at the tested loci, PCR product abundance from the $2 \mathrm{U} \mathrm{ml}^{-1}$ DNase I-treated samples was normalized to that from $0.05 \mathrm{U} \mathrm{ml}^{-1}$-treated samples. The results were then expressed 
relative to GAPDH $\alpha$ and ACTIN-7. The experiment was performed in three biological replicates.

DNase I-chip. We established DNase I hypo-sensitivity profiling based on a published method ${ }^{52}$. Crude nuclei extracts were produced by treating $100 \mathrm{mg}$ of ground frozen plant material in modified nuclei extraction buffer $(20 \mathrm{mM}$ PIPES-KOH pH 7.6, $1 \mathrm{M}$ hexylene glycol, $10 \mathrm{mM} \mathrm{MgCl}, 1 \mathrm{mM}$ ethylene glycol tetraacetic acid (EGTA), $15 \mathrm{mM} \mathrm{NaCl}, 0.5 \mathrm{mM}$ spermidine, $0.15 \mathrm{mM}$ spermine, $0.5 \%$ Triton-X100, $5 \mathrm{mM} \beta$-mercaptoethanol and EDTA-free protease inhibitor cocktail (Roche, Switzerland)) for $15 \mathrm{~min}$ at $4{ }^{\circ} \mathrm{C}$. The homogenate was filtered through Miracloth (Calbiochem, Germany), and a pellet was collected by a 5 -min centrifugation at $1,500 \mathrm{~g}$ at $4{ }^{\circ} \mathrm{C}$. Isolated nuclei were washed once in nuclei washing buffer (nuclei washing buffer; $40 \mathrm{mM}$ Tris- $\mathrm{HCl} \mathrm{pH} \mathrm{8,} 0.3 \mathrm{M}$ sucrose, $10 \mathrm{mM} \mathrm{MgSO}_{4}$ and EDTA-free protease inhibitor cocktail (Roche)) and were used for DNase I (Promega, Wisconsin) digestion for $15 \mathrm{~min}$ (final concentration $4.5 \mathrm{U} \mathrm{ml}^{-1}$ for treatment and $0 \mathrm{U} \mathrm{ml}^{-1}$ for background control samples) at $30^{\circ} \mathrm{C}$ in digestion buffer (nuclei washing buffer plus $1 \mathrm{mM} \mathrm{CaCl}_{2}$ ). The reaction was stopped with $50 \mathrm{mM}$ EDTA. The digested nuclei mixture was treated with Proteinase K (Sigma-Aldrich, Missouri) and DNA was extracted using careful phenol-chloroform extraction and ethanol/salt precipitation. Recovered DNA was redissolved and resolved on agarose gels. Gel slabs containing DNA fragments of sizes above $17 \mathrm{~kb}$ were excised, and DNA was extracted. The extracted DNA was amplified and labelled using the BioPrime DNA Labelling System (Invitrogen, California). Labelled DNA was hybridized to Affymetrix AGRONOMICS1 Arabidopsis tiling arrays (Affymetrix, Santa Clara, CA) as described ${ }^{53}$. The experiment was performed in three biological replicates.

DNase I-chip data analysis. All analysis was performed in $\mathrm{R}^{54}$. DNase I-chip data were normalized with Model-based Analysis of Tiling-arrays (MAT) ${ }^{55}$ implemented in the Aroma Affymetrix package ${ }^{56}$ with the window size parameter set to 500. Inaccessible regions were defined as continuous runs of probes with a MAT score of at least three and were selected using the package Bayesian Analysis of ChIP-chip experiments (BAC) ${ }^{57}$ with minRun and maxGap parameters set to 5,000 and 200, respectively. Hyper-accessible regions were identified similarly as continuous runs of probes with a MAT score of not $>-8$ and with minRun and maxGap parameters set to 100 and 200, respectively. Genomic features such as genes and TEs were considered to be part of an enriched region when the region covered at least half of the feature's length. A gene-specific MAT score was defined as the trimmed mean (trimming the 5\% most extreme values) of all probe-specific MAT scores for the probes located entirely within the transcribed region of a gene. Only genes interrogated by at least three-specific probes were considered.

Bioinformatic analysis. Visualization of tiling array data was done using the Integrated Genome Browser ${ }^{58}$. H3K27me3, H3K9me2 and DNA methylation data were from published results ${ }^{8,53,59}$. All $P$-values for differences between median values of gene sets were calculated using Wilcoxon's-signed rank test.

Transcript profiling. RNA was extracted, labelled and hybridized to Affymetrix Arabidopsis AGRONOMICS1 microarrays as described ${ }^{60}$ using the GeneChip Whole Transcript Sense Target Labelling Assay (Affymetrix). Summarized gene transcript abundances were derived using the Robust Multi-array Average (RMA) algorithm as described ${ }^{53}$. Summarized gene antisense transcript abundances were derived similarly, using the probes from the antisense strand ${ }^{60}$. Strand-specific RNA-seq data were obtained from Gene Expression Omnibus (GEO) (accession code GSM277612 (ref. 41)). Counts of reads with perfect matches to the antisense strand between transcriptional start and end of annotated genes were normalized to gene lengths.

For gene expression profiling by reverse transcription-quantitative PCR, total RNA was extracted from 14-day old seedlings using ISOL-RNA Lysis reagent (5 Prime, Germany) and depleted for genomic DNA using RQ1 RNase free DNase I (Fermentas). First strand cDNA was synthesized using RevertAid First Strand cDNA Synthesis Kit (Fermentas, Germany). Transcript abundance was detected using quantitative PCR with TaqMan Gene Expression Master Mix (Life Technologies, California) and probes from the Universal Probe Library (Roche, Switzerland). Oligonucleotide sequences are listed in Supplementary Table S1. Transcript abundance of genes was normalized to that of GAPDHa and ACTIN-7. The experiment was performed in three biological replicates.

\section{References}

1. Li, B., Carey, M. \& Workman, J. L. The role of chromatin during transcription. Cell 128, 707-719 (2007).

2. Campos, E. I. \& Reinberg, D. Histones: annotating chromatin. Annu. Rev. Genet. 43, 559-599 (2009).

3. Selth, L. A., Sigurdsson, S. \& Svejstrup, J. Q. Transcript elongation by RNA polymerase II. Annu. Rev. Biochem. 79, 271-293 (2010).

4. Grewal, S. I. \& Jia, S. Heterochromatin revisited. Nat. Rev. Genet. 8, 35-46 (2007).
5. Hennig, L. \& Derkacheva, M. Diversity of polycomb group complexes in plants: same rules, different players? Trends Genet. 25, 414-423 (2009).

6. Margueron, R. \& Reinberg, D. The polycomb complex PRC2 and its mark in life. Nature 469, 343-349 (2011).

7. Zhang, X. et al. Whole-genome analysis of Histone $\mathrm{H} 3$ Lysine 27 trimethylation in Arabidopsis. PLoS Biol. 5, 1026-1035 (2007).

8. Oh, S., Park, S. \& van Nocker, S. Genic and global functions for Paf1C in chromatin modification and gene expression in Arabidopsis. PLoS Genet. 4, e1000077 (2008).

9. Weinhofer, I., Hehenberger, E., Roszak, P., Hennig, L. \& Köhler, C. H3K27me3 profiling of the endosperm implies exclusion of polycomb group protein targeting by DNA methylation. PLoS Genet. 6, e1001152 (2010).

10. Cheutin, T. et al. Maintenance of stable heterochromatin domains by dynamic HP1 binding. Science 299, 721-725 (2003).

11. Cheutin, T., Gorski, S. A., May, K. M., Singh, P. B. \& Misteli, T. In vivo dynamics of Swi6 in yeast: evidence for a stochastic model of heterochromatin. Mol. Cell. Biol. 24, 3157-3167 (2004).

12. Festenstein, R. et al. Modulation of heterochromatin protein 1 dynamics in primary mammalian cells. Science 299, 719-721 (2003).

13. Verschure, P. J. et al. Condensed chromatin domains in the mammalian nucleus are accessible to large macromolecules. EMBO Rep. 4, 861-866 (2003).

14. Gorisch, S. M., Lichter, P. \& Rippe, K. Mobility of multi-subunit complexes in the nucleus: accessibility and dynamics of chromatin subcompartments. Histochem. Cell Biol. 123, 217-228 (2005).

15. Bell, O. et al. Accessibility of the Drosophila genome discriminates PcG repression, H4K16 acetylation and replication timing. Nat. Struct. Mol. Biol. 17, 894-900 (2010).

16. Misteli, T. Beyond the sequence: cellular organization of genome function. Cell 128, 787-800 (2007).

17. Fransz, P. \& de Jong, H. From nucleosome to chromosome: a dynamic organization of genetic information. Plant J. 66, 4-17 (2011).

18. Arabidopsis Genome Initiative. Analysis of the genome sequence of the flowering plant Arabidopsis thaliana. Nature 408, 796-815 (2000).

19. Soppe, W. J. et al. DNA methylation controls histone H3 lysine 9 methylation and heterochromatin assembly in Arabidopsis. EMBO J. 21, 6549-6559 (2002).

20. Schönrock, N., Exner, V., Probst, A., Gruissem, W. \& Hennig, L. Functional genomic analysis of CAF-1 mutants in Arabidopsis thaliana. J. Biol. Chem. 281, 9560-9568 (2006).

21. Fransz, P. F. et al. Integrated cytogenetic map of chromosome arm $4 \mathrm{~s}$ of $A$ thaliana: structural organization of heterochromatic knob and centromere region. Cell 100, 367-376 (2000).

22. Fransz, P., Jong, J. H. D., Lysak, M., Castiglione, M. R. \& Schubert, I. Interphase chromosomes in Arabidopsis are organized as well defined chromocenters from which euchromatin loops emanate. Proc. Natl Acad. Sci. USA 99, 14584-14589 (2002).

23. Sperling, K. et al. DNase I sensitivity in facultative and constitutive heterochromatin. Chromosoma 93, 38-42 (1985).

24. Gendrel, A. V., Lippman, Z., Yordan, C., Colot, V. \& Martienssen, R. A. Dependence of heterochromatic histone $\mathrm{H} 3$ methylation patterns on the Arabidopsis gene. DDM1. Science 297, 1871-1873 (2002).

25. Jackson, J. P., Lindroth, A. M., Cao, X. \& Jacobsen, S. E. Control of CpNpG DNA methylation by the KRYPTONITE histone H3 methyltransferase. Nature 416, 556-560 (2002).

26. Weil, M. R., Widlak, P., Minna, J. D. \& Garner, H. R. Global survey of chromatin accessibility using DNA microarrays. Genome Res. 14, 1374-1381 (2004).

27. Eskeland, R. et al. Ring1B compacts chromatin structure and represses gene expression independent of histone ubiquitination. Mol. Cell. 38, 452-464 (2010).

28. Bantignies, F. et al. Polycomb-dependent regulatory contacts between distant Hox loci in Drosophila. Cell 144, 214-226 (2011).

29. Naughton, C., Sproul, D., Hamilton, C. \& Gilbert, N. Analysis of active and inactive $\mathrm{X}$ chromosome architecture reveals the independent organization of 30 $\mathrm{nm}$ and large-scale chromatin structures. Mol. Cell. 40, 397-409 (2010).

30. Mathieu, O., Probst, A. V. \& Paszkowski, J. Distinct regulation of histone H3 methylation at lysines 27 and 9 by CpG methylation in Arabidopsis. EMBO J. 24, 2783-2791 (2005).

31. Tran, R. K. et al. DNA methylation profiling identifies CG methylation clusters in Arabidopsis genes. Curr. Biol. 15, 154-159 (2005).

32. Zhang, X. et al. Genome-wide high-resolution mapping and functional analysis of DNA methylation in Arabidopsis. Cell 126, 1189-1201 (2006).

33. Zilberman, D., Gehring, M., Tran, R. K., Ballinger, T. \& Henikoff, S. Genomewide analysis of Arabidopsis thaliana DNA methylation uncovers an interdependence between methylation and transcription. Nat. Genet. 39, 61-69 (2007).

34. Lyko, F., Ramsahoye, B. H. \& Jaenisch, R. DNA methylation in Drosophila melanogaster. Nature 408, 538-540 (2000). 
35. Baubec, T., Pecinka, A., Rozhon, W. \& Scheid, O. M. Effective, homogeneous and transient interference with cytosine methylation in plant genomic DNA by zebularine. Plant J. 57, 542-554 (2008).

36. Mathieu, O., Reinders, J., Caikovski, M., Smathajitt, C. \& Paszkowski, J. Transgenerational stability of the Arabidopsis epigenome is coordinated by CG methylation. Cell 130, 851-862 (2007).

37. Jones, P. A. \& Taylor, S. M. Cellular differentiation, cytidine analogs and DNA methylation. Cell 20, 85-93 (1980).

38. Vongs, A., Kakutani, T., Martienssen, R. A. \& Richards, E. J. Arabidopsis thaliana DNA methylation mutants. Science 260, 1926-1928 (1993).

39. Saze, H., Scheid, O. M. \& Paszkowski, J. Maintenance of cpg methylation is essential for epigenetic inheritance during plant gametogenesis. Nat. Genet. 34, 65-69 (2003).

40. Kakutani, T., Munakata, K., Richards, E. J. \& Hirochika, H. Meiotically and mitotically stable inheritance of DNA hypomethylation induced by $d d m 1$ mutation of Arabidopsis thaliana. Genetics 151, 831-838 (1999).

41. Lister, R. et al. Highly integrated single-base resolution maps of the epigenome in Arabidopsis. Cell 133, 523-536 (2008).

42. Henikoff, S. \& Shilatifard, A. Histone modification: cause or cog? Trends Genet. 27, 389-396 (2011).

43. Volpe, T. A. et al. Regulation of heterochromatic silencing and histone $\mathrm{H} 3$ lysine-9 methylation by RNAi. Science 297, 1833-1837 (2002).

44. Chen, E. S. et al. Cell cycle control of centromeric repeat transcription and heterochromatin assembly. Nature 451, 734-737 (2008).

45. Lu, J. \& Gilbert, D. M. Proliferation-dependent and cell cycle regulated transcription of mouse pericentric heterochromatin. J. Cell. Biol. 179, 411-421 (2007).

46. Kloc, A., Zaratiegui, M., Nora, E. \& Martienssen, R. RNA interference guides histone modification during the $\mathrm{S}$ phase of chromosomal replication. Curr. Biol. 18, 490-495 (2008)

47. Bantignies, F., Grimaud, C., Lavrov, S., Gabut, M. \& Cavalli, G. Inheritance of Polycomb-dependent chromosomal interactions in Drosophila. Genes Dev. 17, 2406-2420 (2003).

48. Francis, N. J., Kingston, R. E. \& Woodcock, C. L. Chromatin compaction by a Polycomb group protein complex. Science 306, 1574-1577 (2004).

49. Lorincz, M. C., Dickerson, D. R., Schmitt, M. \& Groudine, M. Intragenic DNA methylation alters chromatin structure and elongation efficiency in mammalian cells. Nat. Struct. Mol. Biol. 11, 1068-1075 (2004).

50. Zilberman, D., Coleman-Derr, D., Ballinger, T. \& Henikoff, S. Histone H2A.Z and DNA methylation are mutually antagonistic chromatin marks. Nature 456, 125-129 (2008)

51. Chang, S. \& Pikaard, C. S. Transcript profiling in Arabidopsis reveals complex responses to global inhibition of DNA methylation and histone deacetylation. J. Biol. Chem. 280, 796-804 (2005).

52. Sabo, P. J. et al. Genome-scale mapping of DNase I sensitivity in vivo using tiling DNA microarrays. Nat. Methods 3, 511-518 (2006).

53. Rehrauer, H. et al. AGRONOMICS1: A new resource for Arabidopsis transcriptome profiling. Plant Physiol. 152, 487-499 (2010).
54. R Development Core Team. R: A Language and Environment for Statistical Computing (R Foundation for Statistical Computing, Vienna, Austria, 2010).

55. Johnson, W. E. et al. Model-based analysis of tiling-arrays for ChIP-chip. Proc Natl Acad. Sci. USA 103, 12457-12462 (2006).

56. Bengtsson, H., Simpson, K., Bullard, J. \& Hansen, K. aroma.affymetrix: A generic framework in $\mathrm{R}$ for analyzing small to very large Affymetrix data sets in bounded memory. Tech Report No.745, Department of Statistics, University of California, Berkeley (2008).

57. Gottardo, R., Li, W., Johnson, W. E. \& Liu, X. S. A flexible and powerful Bayesian hierarchical model for ChIP-Chip experiments. Biometrics 64, 468-478 (2008).

58. Nicol, J. W., Helt, G. A., Blanchard, Jr S. G., Raja, A. \& Loraine, A. E. The Integrated Genome Browser: free software for distribution and exploration of genome-scale datasets. Bioinformatics 25, 2730-2731 (2009).

59. Hsieh, T. F. et al. Genome-wide demethylation of Arabidopsis endosperm. Science 324, 1451-1454 (2009).

60. Müller, M., Patrignani, A., Rehrauer, H., Gruissem, W. \& Hennig, L. Evaluation of alternative RNA labeling protocols for transcript profiling with Arabidopsis AGRONOMICS1 tiling arrays. Plant Meth. 8, 18 (2012).

\section{Acknowledgements}

We thank Jonathan Seguin for help with the bioinformatics analysis, Benjamin Knoerlein for sharing his experience in data processing, and Dr Markus Kalisch for his consultation in statistics. We thank the Functional Genomics Centre Zurich for microarray hybridisation and scanning. This work was supported by the Sixth Framework Program of the European Commission through the AGRON-OMICS Integrated Project (LSHG-CT2006-037704), by the Swedish Research Council and by the Swiss National Science Foundation (31003A_130272 and 31003A_132971).

\section{Author contributions}

L.H. and H.S. conceived the study. H.S. and T.W. performed experiments. H.S., L.H. and A.S. analysed data. H.S., L.H. and W.G. wrote the manuscript.

\section{Additional information}

Accession codes: Microarray data have been deposited in ArrayExpress under accession code E-TABM-1219.

Supplementary Information accompanies this paper at http://www.nature.com/ naturecommunications

Competing financial interests: The authors declare no competing financial interests.

Reprints and permission information is available online at http://npg.nature.com/ reprintsandpermissions/

How to cite this article: Shu H. et al. Distinct modes of DNA accessibility in plant chromatin. Nat. Commun. 3:1281 doi: 10.1038/ncomms2259 (2012). 